\title{
Massive gastric dilatation: the radiological picture of eating disorder
}

\author{
Federico Pasin ${ }^{1}$ (D) Antonio Modoni ${ }^{2} \cdot$ Lorenzo Teti $^{3} \cdot$ Luca Rosi $^{4}$ • \\ Giorgio Ragni $^{1}$
}

Received: 3 March 2016/Accepted: 19 March 2016/Published online: 4 April 2016

(c) SIMI 2016

\begin{abstract}
A 23-year-old man with no past medical history presented to the emergency department(ED) with severe, diffuse abdominal pain, and nausea. He reported a binge eating several hours before the onset of symptoms. He had been unable to induce vomiting to reach relief at home. He reported that he was in his usual state of health until the heavy food intake. Physical examination revealed a markedly distended abdomen, and epigastric tenderness with absent bowel sounds. There was no rebound, and no masses were palpable. The remainder of the physical examination was unremarkable. Temperature was $37^{\circ} \mathrm{C}$, heart rate: 95 beats per minute, blood pressure $105 / 75 \mathrm{mmHg}$, respiratory rate 32 breaths per minute, and body mass index was 22 . Laboratory examinations showed a mild leukocytosis, amylase: $90 \mathrm{U} / \mathrm{L}$, and in the normal range: electrolytes, acid-base, and iron profile, and the hemoglobin value. An abdominal plain film showed a large gastric bubble and a paucity of small bowel gas without evidence of free abdominal air (Fig. 1). Computed
\end{abstract}

Federico Pasin

f.pasin@ospedale.cremona.it

1 Internal Medicine Unit, Istituti Ospitalieri di Cremona/ Presidio Ospedaliero Oglio-Po, Via Staffolo 51, 26041 Casalmaggiore, Italy

2 Radiological Unit, Istituti Ospitalieri di Cremona/Presidio Ospedaliero Oglio-Po, Casalmaggiore, Italy

3 Emergency Unit, Istituti Ospitalieri di Cremona/Presidio Ospedaliero Oglio-Po, Casalmaggiore, Italy

4 Psychiatric Unit, Istituti Ospitalieri di Cremona/Presidio Ospedaliero Oglio-Po, Casalmaggiore, Italy tomography of abdomen and pelvis showed massive gastric distension with a considerable mass effect on adjacent organs by fluid and food resulting in gastric obstruction without perforation (Figs. 2, 3, 4). A nasogastric tube was placed, and a large amount of gastric contents consisting of partially digested particulate material were evacuated during 3 days (10 1) with partial relief of symptoms. Subsequently, after 3 days, he underwent radiography with contrast medium that showed delayed gastric emptying (Fig. 5), and an endoscopic study showed no pathological signs in the esophageal and gastric mucosa. Even in the context of normal body weight and no previously known eating disorder, the massive gastric distension following a "reported" single eating binge associated with subsequent delayed gastric emptying, raised the high probability of a severe eating disorder, probably bulimia, at the first clinical and radiological presentation. Afterward, despite a thorough medical history assessment regarding, in particular the nutritional aspect, the patient continued to deny previous eating disorders; nor was there any other helpful information reported by the parents in this regard. Furthermore, a repeated careful examination did not reveal any dental changes: there was no erosion of tooth enamel, nor small hemorrhages of palate, nor gland salivary swelling. The early stage of the eating disorder or an oral "obsessive" care may have been the explanation of the absence of these pathognomonic aspects. The patient underwent a complete detailed psychiatric evaluation for conclusive diagnostic definition. Recognition of such complications is critical to effective patient care, and requires a radiologist to be aware of the spectrum of imaging abnormalities that may be seen. Many patients are reluctant to disclose their condition, so radiological findings have a central role in identifying undiagnosed eating disorders [1-3] . 


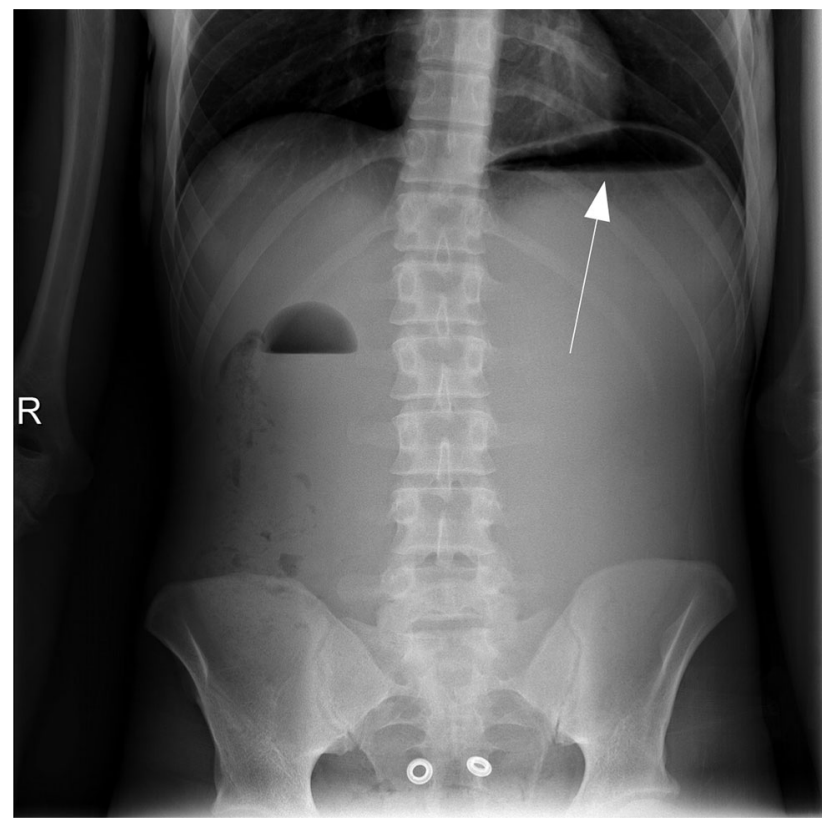

Fig. 1 Plain film X-ray upon admission. A huge gastric dilatation with a high fluid level (white arrow)

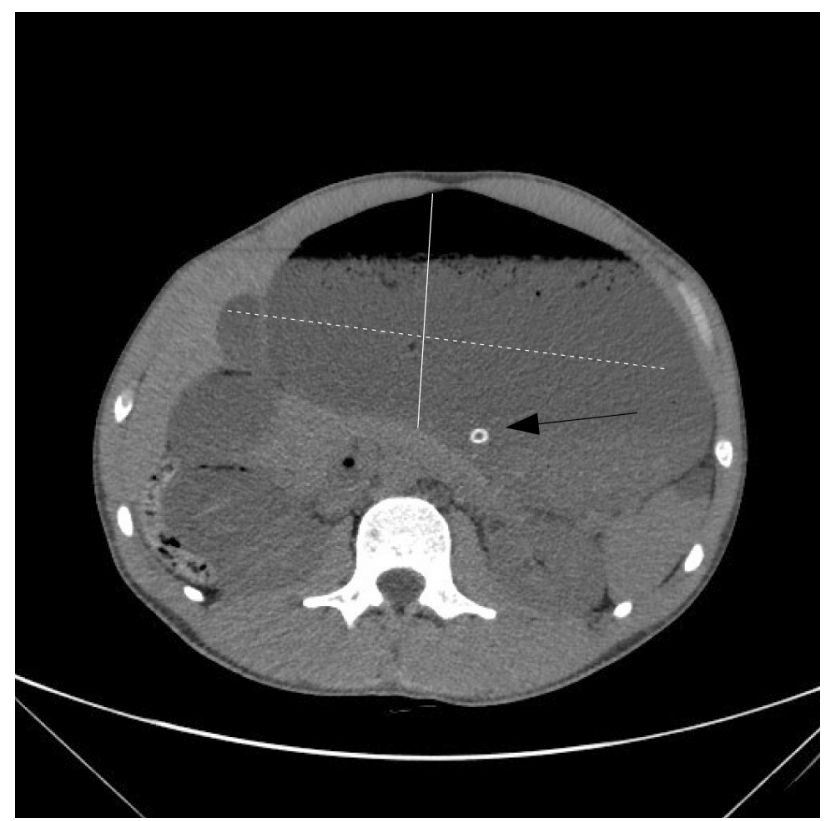

Fig. 2 Abdominopelvic computed scan (axial). Massive gastric dilatation (white lines) occupied the entire abdominal cavity from the diaphragm to the pelvis with a large volume of food inside the stomach, displacement, and compression of other peritoneal and retroperitoneal organs. Nasogastric tube (black arrow)

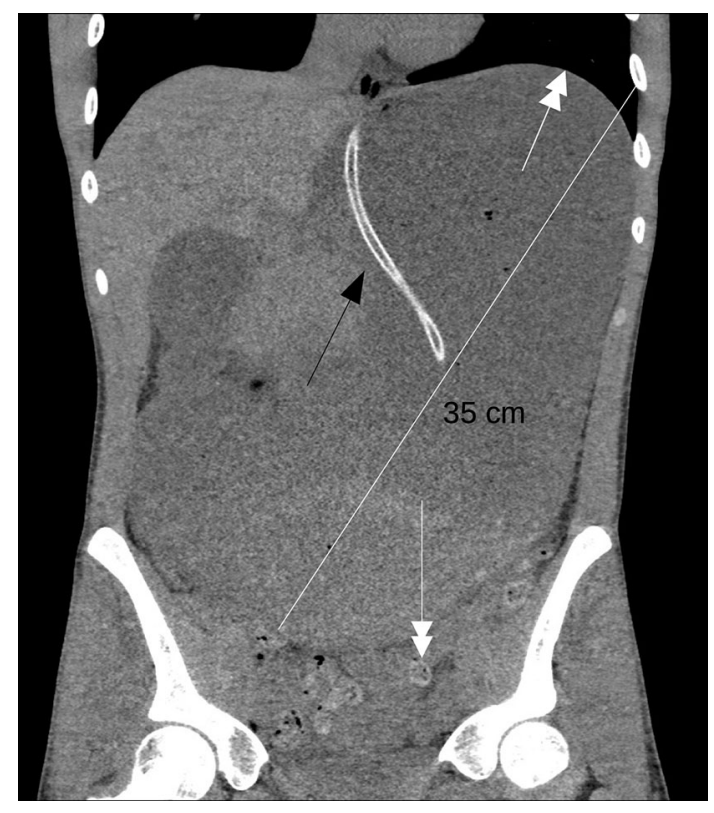

Fig. 3 Abdominopelvic computed scan (coronal). Massive gastric dilatation (white lines) occupied the entire abdominal cavity from the diaphragm to the pelvis with a large volume of food inside the stomach, displacement and compression of other peritoneal and retroperitoneal organs (double headed arrow). Nasogastric tube (black arrow)

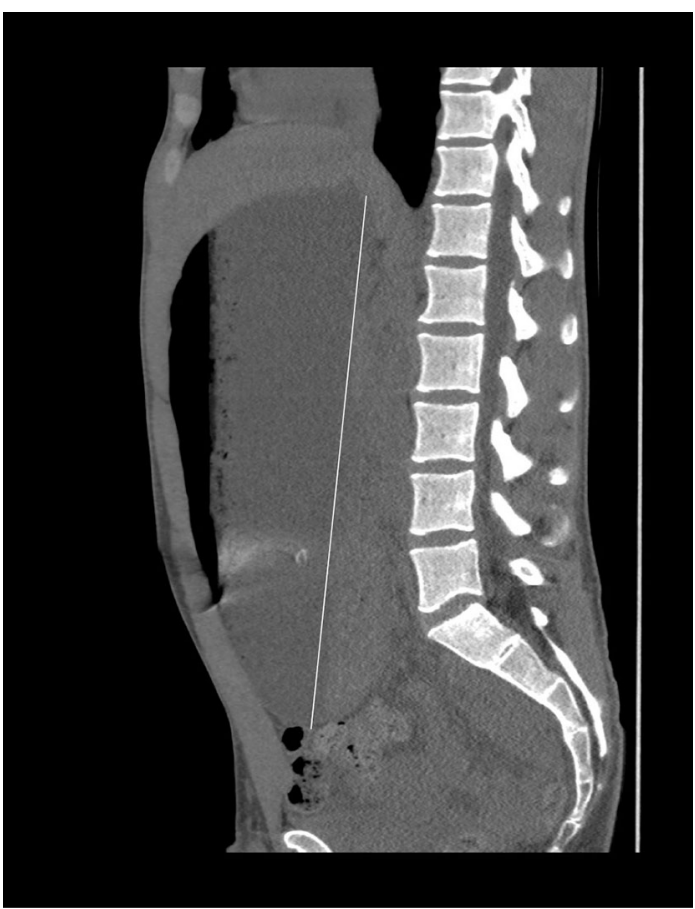

Fig. 4 Abdominopelvic computed scan (sagittal projection). Massive gastric dilatation occupied the entire abdominal cavity from the diaphragm to the pelvis with a large volume of food inside the stomach, displacement and compression of other peritoneal and retroperitoneal organs 


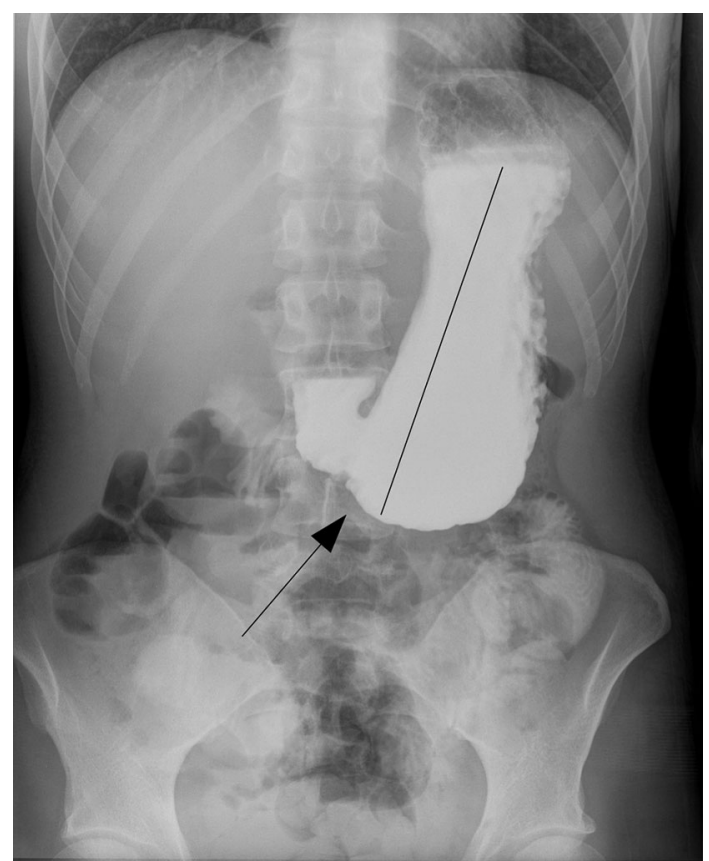

Fig. 5 X-ray with barium swallow. After gastric decompression, mild indirect signs of gastric atony (black arrow)

\section{Compliance with ethical standards}

Conflict of interest The authors declare that they have no conflict of interest.

Statement of human and animal rights All procedures performed in studies involving human participants were in accordance with the ethical standards of the institutional and/or national research committee and with the 1964 Helsinki declaration and its later amendments or comparable ethical standards.

Informed consent Informed consent was obtained from all individual participants included in the study.

\section{References}

1. Bowden DJ, Kilburn-Toppin F, Scoffings DJ (2013) Radiology of eating disorders: a pictorial review. Radiographics 33(4): $1171-1193$

2. Sato Y, Fukudo S (2015) Gastrointestinal symptoms and disorders in patients with eating disorders. Clin $\mathbf{J}$ Gastroenterol $8(5): 255-263$

3. Czarnecki CA, O'Coclain DF (2002) Images in clinical medicine. Severe abdominal pain in a girl. N Engl J Med 347(10):e3 